\title{
LOS PROCESOS MORFOLÓGICOS QUE AFECTAN LOS VERBOS IRREGULARES EN ESPAÑOL
}

\author{
Flor María Garita H.
}

\begin{abstract}
RESUMEN
El presente trabajo tiene por objeto estudiar las irregularidades de los verbos en español a la luz de los planteamientos teóricos sobre el estudio de la morfología de Hockett y P.H. Matthews. Antes se hará un recorrido sobre algunas posiciones, principalmente, el enfoque de la Real Academia Española y otros exponentes de la gramática.
\end{abstract}

\begin{abstract}
This article deals with the irregularities of Spanish verbe in the light of Hokett and P.H. Matthews' porposals of the study of morphology. Before that, we will look at some views, especially the approach of the Real Academia Española and other exponents of grammar.
\end{abstract}

\section{Los verbos irregulares}

La Real Academia Española en Esbozo de una nueva gramática de la lengua española (1986) plantea que "las irregularidades que se presentan en la conjugación española, con raras excepciones de las que hablaremos más adelante, afectan la raíz verbal... En su conjunto, las irregularidades son resultado de la acción de las leyes fonéticas sobre el sistema entero de la lengua española" (270); por tanto, el estudio es puramente formal.

Para describir una irregularidad determinada hay que tener en cuenta dos series de datos: los que se refieren a su estructura fonológica y los que se refieren a su extensión (formas flexivas, conjugaciones y verbos en que aparece). La irregularidad puede ser vocálica, consonántica o mixta. Hay irregularidades que afectan al tema o al tema y a la raíz simultáneamente. Hay otras irregularidades de carácter excepcional tales como contracciones, o verbos con más de una raíz, futuros y condicionales irregulares, participios y gerundios irregulares.

Alcina F. y Blecua R. en Gramática Española (1975) en una descripción estructural, entendida la lengua como un sistema de signos, indican que "las irregularidades se inscriben dentro del ámbito de los inventarios de alomorfos de un morfema o como casos de supletivismo. Aparte de los casos de supletivismo se distinguen dos clases particulares de alternancia según se produzcan en el morfema lexemático o en este y los morfemas gramaticales" (7689). De esta forma proponen un inventario de los tipos de alternancias más representativos: 
1 Alternancias vocálicas en el lexema: verbos como entender, entiendo; morir, muero; dormir, durmió etc.

2. Variaciones consonánticas en el lexema: verbos como salir, salgo; caer, caigan; huir, huya.

3. Alternancia vocálica y variación consonántica: en verbos como decir, digan; nacer, nazca.

4. Formas atemáticas del grupo de futuro: poner, pondrán, saber, sabrían.

5 Presentes agudos monosilábicos: hacer, haz; decir, di; venir, ven.

6 Los pretéritos fuertes: tener, tuve; caber, cupe.

7. Verbos defectivos: Es otra irregularidad por no estar en uso determinadas formas verbales.

En realidad, estos autores no examinan exaustivamente la alternancia de alomorfos, sino que se limitan a presentar las irregularidades en forma muy parecida a como lo hace la Academia Española.

\section{Propuesta de Matthews}

Matthews, con una perspectiva también estructuralista, enfoca la teoría de la morfología sincrónica desde tres ángulos: se deben establecer las unidades básicas, señalar la realización de estas unidades y establecer los criterios que permiten determinar el análisis morfológico de cualquier lengua. Hockett, por su parte, distingue tres enfoques muy generales de la estructura lingüística, a saber: Elemento y colocación, Elemento y proceso y Palabra- paradigma.

En el primer enfoque, Elemento y colocación, los segmentos concurrentes se denominan morfemas y la relación entre ellos es de simple sucesividad. Por ejemplo, en un verbo regular como amar, en pretérito imperfecto de indicativo, "amábamos" tendríamos los siguientes morfemas: am- que es la raíz, -a la vocal temática,- ba morfema de tiempo y modo, y -mos, morfema de persona número.

En el enfoque Palabra-paradigma, las unidades básicas se conciben como abstracciones de palabras tomadas en su totalidad y no como abstracciones de segmentos aislados. Se puede decir que a las palabras se les considera exponentes de los diversos miembros del paradigma.

En Elemento y proceso, enfoque que se utiliza en este análisis, las unidades básicas son los morfemas y, a diferencia de los anteriores, las relaciones entre los morfemas son secuenciales.

Este análisis se centra en los procesos fonológicos o cambios que sufren las palabras en su estructura fonológica. Se les llama morfofonológicos o morfofonémicos. En cuanto a la realización, se pueden distinguir tres tipos sucesivos de reglas:

1. La mayor parte de los morfemas dispondrían, desde el principio, de algún tipo de configuración básica fonológica, es decir, se debe determinar la forma fonológica de las unidades mínimas.

2. Unos pocos morfemas tendrían la capacidad de modificar a otros morfemas vecinos de determinadas maneras.

3. Hay otro conjunto de procesos que harían que las representaciones básicas resultantes de 1 y 2 interactuaran entre sí. Se debe proponer una forma básica. 
La ventaja de este procedimiento es que permite describir tanto estructuras discretas, como las de los verbos regulares, como las no discretas de los verbos irregulares del español.

Los procesos morfológicos consisten en derivar formas léxicas a partir de otras formas léxicas. De esta manera, debe establecerse la forma básica a partir de la cual se derivaría la forma resultante. Así, en un verbo irregular en estudio, tomaré como forma base la raíz del infinitivo y, a partir de aquí, se analizarán los procesos que sufre cada verbo.

\section{Tipología de los procesos}

\subsection{Operación de identidad}

Es una operación cuya entrada es idéntica a la salida. En el proceso, dos elementos morfológicamente separados reaccionan para tener una única realización. Matthews encuentra un paralelo con la teoría del sandhi, proceso fonológico en el que los sonidos sufren cambios por influencia de otros sonidos vecinos, aunque producidos por fenómenos fonológicos diferentes. Así el presente o el pretérito del verbo morir se deriva de la raíz del infinitivo mor, la cual sufre un proceso de reemplazamiento de vocales y da muero y murió respectivamente. En el primer caso, la vocal 'o' de la raíz se diptonga por influencia del acento.

Matthews establece tres elementos que participan en el proceso:

Derivando: Es la forma resultante después de aplicar el proceso

Operando: Es la forma inicial a la que se aplica el proceso, en nuestro caso, la raíz del infinitivo.

Formativo: el elemento que se reemplaza, se suprime, se adiciona o se sustituye.

Los procesos que intervienen en los verbos irregulares del español son el reemplazamiento, la adición y la sustracción. Seguidamente se define cada proceso.

\subsection{Proceso de Reemplazamiento}

Consiste en reemplazar fonemas del operando o raíz, motivado por la presencia de fonemas vecinos o de suprasegmentales como el acento. La modificación puede ser parcial o total. Cuando es total, recibe el nombre de supleción.

Matthews agrega que las posibles subdivisiones de este tipo son muy numerosas y pueden distinguirse efectivamente por criterio fonéticos al igual que por criterios puramente lógicos. Algunos se emplean en tipos concretos de operaciones y en grupos o lenguas determinados.

Entre los reemplazamientos más comunes en los verbos irregulares tenemos cambio vocálico, cambio consonántico o cambio mixto, es decir, simultáneamente cambian las vocales y las consonantes. Por ejemplo, en los siguientes verbos:

$\begin{array}{llll}\text { Operando } & \text { Formativo } & \text { Derivando } & \text { Reemplazamiento } \\ \text { Morir } & \mathrm{o}>\mathrm{ue} & \text { muero } & \text { vocálico } \\ \text { Hacer } & \mathrm{c}>\mathrm{g} & \text { hago } & \text { consonántico } \\ \text { Caber } & \mathrm{ab}>\text { up } & \text { cupe } & \text { mixto }\end{array}$




\subsection{Proceso de Adición}

Es un proceso que consiste en agregar fonemas al operando o raíz. En el proceso se pueden adicionar consonantes o vocales y consonantes simultáneamente. Por ejemplo:

$\begin{array}{llll}\text { Traer } & \text { adición de } \mathrm{j} & \text { trajo } & \text { consonante } \\ \text { Traer } & \text { adición de ig } & \text { traiga } & \text { mixto } \\ \text { Salir } & \text { adición de d } & \text { saldré } & \text { consonante }\end{array}$

\subsection{Proceso de Sustracción}

Consiste en sustraer fonemas de la raíz. Este proceso se da al construir el futuro y el condicional simples de ciertos verbos irregulares en español. Por ejemplo, en los verbos salir y tener.

En el verbo salir, el futuro regular sería saliré. En el operando se dan dos procesos. Primero se pierde la vocal temática 'i' (se sustrae) y luego se agrega la consonante 'd'. Ambos fenómenos se dan en el futuro y en el condicional fenómenos.

Seguidamente se presenta un desglose de los principales verbos que presentan estos

\section{Procesos}

\section{Reemplazamiento}

1.1. Vocálico.

$\begin{array}{llll} & \text { Vocales } & + & \text { Cerradas } \\ \text { e/i } & \text { pedir } & \rightarrow & \text { pidió } \\ & \text { medir } & \rightarrow & \text { midió } \\ \text { sentir } & \rightarrow & \text { sintió } \\ \text { morir } & \rightarrow & \text { murió } \\ \text { o/u } & \text { dormir } & \rightarrow & \text { durmió } \\ & \text { Diptongos } & & \\ & \text { mover } & \rightarrow & \text { muevo } \\ \text { o/ue } & \text { dormir } & \rightarrow & \text { duermo } \\ & \text { volver } & \rightarrow & \text { vuelvo } \\ & \text { tener } & \rightarrow & \text { tiene } \\ \text { e/ie } & \text { querer } & \rightarrow & \text { quiere } \\ & \text { venir } & \rightarrow & \text { viene } \\ & \text { adquirir } & \rightarrow & \text { adquiere } \\ \text { i/ie } & \text { inquirir } & \rightarrow & \text { inquiere }\end{array}$


u/ue jugar $\rightarrow$ juego

1.2. Consonántico (se reemplazan consonantes)

- Una consonante.

$\mathrm{c} / \mathrm{g}$ hacer $\rightarrow$ haga

b/y haber $\rightarrow$ haya

- $\quad$ Mixto (vocales y consonantes)

ec/ig decir $\rightarrow$ digo

ab/ep caber $\rightarrow$ quepo

ab/up saber $\rightarrow$ supe

ec/ij decir $\rightarrow$ dijo

1.3. Supleción.

Ir — voy -iba-fui-iré

Ser — soy-era-fui-seré

\section{Proceso aditivo}

a) Se agrega una consonante.

$\begin{array}{llll}\text { "z" } & \text { nacer } & \rightarrow & \text { nazco } \\ \text { traer } & \rightarrow & \text { traje } \\ \text { salir } & \rightarrow & \text { salgo } \\ \text { "g" } & \text { venir } & \rightarrow & \text { vengo } \\ \text { poner } & \rightarrow & \text { pongo } \\ \text { huir } & \rightarrow & \begin{array}{l}\text { huyo (presente indicativo) } \\ \text { huya (presente subjuntivo) } \\ \text { huye tú (imperativo) } \\ \text { oye (presente indicativo) } \\ \text { oye tú (imperativo) }\end{array} \\ & & & \end{array}$

b) Se agrega vocal y consonante.

$\begin{array}{lll}\text { "ig" } & \rightarrow & \text { traigo } \\ \text { caer } & \rightarrow & \text { caigo } \\ \text { oír } & \rightarrow & \text { oigo } \\ \text { roer } & \rightarrow & \text { roigo }\end{array}$

\section{Proceso de Sustracción}

3.1 Sustracción de vocal temática

Caber $\rightarrow$ cabré-cabría 


\begin{tabular}{|c|c|}
\hline Haber - & $\rightarrow$ \\
\hline Querer - & \\
\hline Saber - & \\
\hline
\end{tabular}

\subsection{Proceso de sustracción de vocal temática y adición “ $d$ ”}

a) Pérdida de "e" y aumento de "d"

Poner $\rightarrow$ pondré $\quad$ Tener $\rightarrow$ tendré $\quad$ Valer $\rightarrow$ valdré

b) Pérdida de "i" y adición de "d"

Salir $\rightarrow$ saldré $\quad$ Venir $\rightarrow \quad$ vendré

Merecen especial atención los verbos que Alcina Franck denomina monosilábicos y los que tanto él como la Academia llaman perfectos fuertes, apoyados en la denominación latina. Tanto unos como otros no son más que muestras de diferentes procesos sufridos durante la conjugación. Veamos algunos casos:

En imperativos y formas del presente de indicativo. En formas como sé y sed de ser; pon de poner; ten de tener; ve e id de ir; ven de venir; haz de hacer; di de decir; da de dar y sal de salir, según Alcina Franck son verbos de morfema léxico sin morfema gramatical. En los casos de soy de ser, doy de dar y voy de ir, los verbos agregan una 'y' que aparece igualmente en el lexema voy del verbo ir y se presentan sin morfema auxiliar, como lexema puro.

No queda claro a qué se refiere con lexema puro o con lexema auxiliar.

Más clara aparece la explicación de los perfectos fuertes cono tuve, conduje, traduje, cupe, estuve, hubo, pude, supe, dijo, tratados como variaciones consonánticas, alternancias vocálicas o adiciones de vocales o consonantes.

En el caso de los verbos en imperativo sé y sed, pon, ten, ven, haz, da y sal no hay irregularidad, aunque son monosilábicos. Sí hay irregularidad en el imperativo ve de ir que, como se dijo antes, sufre supleción; pero id conserva la raíz i . En el caso de de di, la raíz del verbo sufre dos procesos: hay un reeplazamiento vocálico de e por i como sucede en el presente y luego una sustración de la consonante c. Un fenómeno parecido presenta el verbo saber en presente de indicativo, primera persona: sé. El verbo sufre reemplazamiento de vocal a por e y sustracción de la consonante. Igualmente sucede con haber, he.

Respecto de los perfectos fuertes, se analizarán individualmente para verificar los procesos que sufren en la siguiente forma.

$\begin{array}{llllll}\text { Forma base } & \text { Forma verbal } & \text { Irregularidad } & \text { Tipo } & \text { Proceso } \\ \text { Decir } & \text { dije } & \mathrm{e}-\mathrm{i} & \mathrm{c}-\mathrm{j} & \text { Voc.-cons. } & \text { Mixto } \\ \text { Tener } & \text { tuve } & \mathrm{e}-\mathrm{u} & \mathrm{n}-\mathrm{v} & \text { Voc.-cons. } & \text { Mixto } \\ \text { Caber } & \text { cupe } & \mathrm{a}-\mathrm{u} & \mathrm{b}-\mathrm{p} & \text { Voc.-cons. } & \text { Mixto } \\ \text { Saber } & \text { supe } & \mathrm{a}-\mathrm{u} & \mathrm{b}-\mathrm{p} & \text { Voc.-cons. } & \text { Mixto } \\ \text { Querer } & \text { quise } & \mathrm{e}-\mathrm{i} & \mathrm{r}-\mathrm{s} & \text { Voc.-cons. } & \text { Mixto }\end{array}$




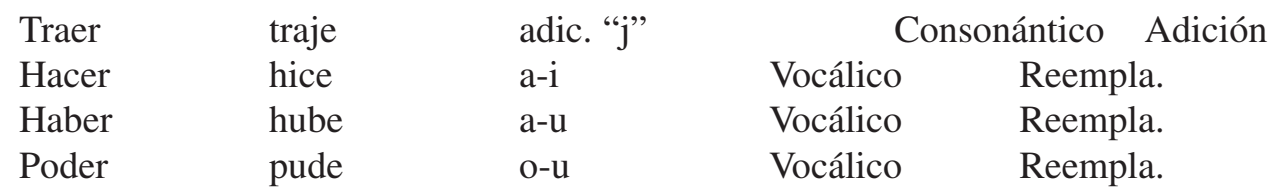

\section{Conclusiones}

El enfoque de los procesos morfológicos de Matthews constituye una herramienta valiosa para el análisis sincrónico de fenómenos morfológicos de una lengua. En este estudio se han explicado las principales irregularidades de los verbos del español aplicando este marco teórico. Cabe aclarar que el estudio no se agota en los verbos, pues las irregularidades, como dice la Real Academia en su Esbozo, "afectan el sistema entero de la lengua española" (pág 270). El enfoque, además de permitir analizar la formas no personales del verbo, podría aplicarse a otras categorías gramaticales que presenten estos fenómenos

\section{Bibiliografía}

Alcina, Franck Juan y José Manuel Blecua. 1975. Gramática Española. Barcelona: Editorial Ariel.

Alcoba, Santiago. 1999. "La flexión verbal” Gramática descriptiva de la lengua española. Madrid: Editorial Espasa.

Real Academia Española. 1974. Esbozo de una nueva gramática de la lengua española. Madrid: Editorial Espasa _ Calpe.

Hokett, Ch. 1971. Curso de lingüística moderna. 3 edición Argentina: Editorial Universitaria.

Lyons, J. 1970. Nuevos horizontes de la lingûística. Madrid: Alianza.

Mathews, P. H. 1980. Morfología: Introducción a la teoría de las estructuras de la palabra. Madrid: Paraninfo. 